\title{
Assessment of Quality of Pediatric Emergency Triage and Its Associated Factors in Wolaita Zone, Ethiopia
}

\author{
Daniel Baza Gargamo ${ }^{1}$, Addishiwet Fantahun ${ }^{2}$, Temesgen Lera Abiso ${ }^{3}$ \\ ${ }^{1}$ Department of Nursing, Wolaita Sodo University, Wolaita Sodo, Ethiopia \\ ${ }^{2}$ Department of Nursing, Addis Ababa University, Addis Ababa, Ethiopia \\ ${ }^{3}$ School of Public Health, Wolaita Sodo University, Wolaita Sodo, Ethiopia
}

Email address:

danielbaza9@gmail.com (D. B. Gargamo), addishiwet.f@gmail.com (A. Fantahun), temelera@gmail.com (T. L. Abiso)

To cite this article:

Daniel Baza Gargamo, Addishiwet Fantahun, Temesgen Lera Abiso. Assessment of Quality of Pediatric Emergency Triage and Its Associated Factors in Wolaita Zone, Ethiopia. American Journal of Health Research. Vol. 7, No. 4, 2019, pp. 123-133. doi: 10.11648/j.sjph.20190704.13

Received: June 1, 2019; Accepted: July 23, 2019; Published: August 29, 2019

\begin{abstract}
Background: Most deaths of children in hospital frequently occur within the first 24 hours of admission. Immediate triage on arrival may prevent many of these deaths. Pediatric emergency triage assessment and treatment was introduced in 2014 in Ethiopia, however, there is no evidence which shows its quality and associated factors in the country. Objective:-This study aimed to assess the quality of pediatric emergency triage and its associated factors in selected hospitals of Wolaita zone 2017. Methods: A facility-based cross-sectional study design was used March to April 2017. A total of 178 health care workers (HCWs) were included in this study. The data was collected by using a self-administered questionnaire on the HCWs, and an observation checklist for hospitals assessment. We performed descriptive and multivariable logistic regression analyses; adjusted odds ratio and $95 \% \mathrm{CI}$ were used to determine statistically significant associations. Results: This study indicated that $41.7 \%$ of HCWs did not correctly define triage, $81 \%$ did not know triage duration, $85.7 \%$ did not identify all triage categories and $64 \%$ did not categorize child with urgent signs. Conclusion: The overall quality of pediatric emergency triage service was poor.
\end{abstract}

Keywords: Quality, Pediatrics, Emergency, Triage, Assessment

\section{Introduction}

Children in sub-Saharan Africa are more than 15 times more likely to die before the age of five than children in developed regions [1] One reason for this may be that many hospitals in low-income countries lack a formal triage system [2]. Clinicians usually see the patients on a "first-come-firstserved" basis rather than their acuity level [3]. Seventy-Five percent of 7.6 million children under five years old who die each year worldwide are in Africa or Asia [4].

Most deaths of children in the hospitals frequently occur within the first 24 hours of admission which is often attributable to easily treatable, time-sensitive illness [2, 5]. In many hospitals, children are not checked before a senior health worker examines them. The lack of immediate triage may then result in death due to a treatable condition [6]. However, this may be mitigated by rapid triage for all children presenting to hospital in order to determine whether any emergency or priority signs are present, and ensuring appropriate emergency treatment [6-8].

Pediatric emergency triage is a categorization of patients according to their level of acuity on arrival at an emergency unit of any hospital without delay [7]. It is an essential function of health care workers, and is critical to the effective management of modern emergency departments. Early triage is an important component of quality and safety within the emergency department [9], and such a process is critical for the identification of ill or injured children who may benefit from early life-saving interventions [10].

Possible barriers to the effective emergency care of children include overcrowded emergency care areas, poor facilities for children, long waiting times for and limited access to hospital beds, poor staff training for pediatric emergency conditions, insufficient or inadequate equipment and supplies, lack of child-specific policies and guidelines, 
and ignorance or acceptance of poorer standards of care for children in the Emergency department (ED) $[4,11,12,14]$.

Even though pediatric emergency triage assessment and treatment was introduced in 2014 in Ethiopia, there is no evidence that shows its quality and associated factors. Therefore, this study was aimed to assess the quality of pediatric emergency triage and its associated factors in Wolaita Zone, South Nation Nationalities and Peoples Region (SNNPR) Ethiopia

\section{Material and Methods}

\subsection{Study Setting and Design}

The study was carried out in Wolaita Zone which is located $334 \mathrm{~km}$ from the capital city of the country, Addis Ababa and $151 \mathrm{~km}$ far from the SNNPR regional city Hawassa. Wolaita zone is one of the 13 zones in the region with a population of $1,928,196$. Considering health infrastructure, The Wolaita zone has 68 functional health centers, 6 hospitals of one teaching and referral, one general, one district hospital and the three primary level hospitals with 341 health posts. The study design was a facility based descriptive cross-sectional study.

\subsection{Sample Size and Sampling Procedure}

The sample size was determined by taking $50 \%$ estimated proportion of pediatric triage since its status was not known. Assuming 5\% margin of error, 95\% confidence level and 5\% non-response rate, the total sample size calculated was 384 . Since the source population was less than 10,000 , a single population proportion correction formula was used and the final sample size calculated was 229. However, in the selected hospitals there were 178 HCWS working in the pediatric Triage units. So, we included the total sample size of 178 .

Sampling Procedure

All hospitals providing emergency triage assessment and treatment were selected and health care professionals working in emergency and pediatric unit were chosen as study population in a deliberative and non-random fashion by purposive sampling technique to achieve the study objective. Units were purposely selected to include all health care workers who have had experience in caring for children with emergency or priority signs. All health care professionals at the selected units or working in the emergency room were involved in the study.

\subsection{Data Collection Procedure}

Data were collected using self-administered questionnaires The data collection tool was adapted from the literature on maternal health surveys $[1,2,6,9,14]$. The tool contained four sections which assessed socio-demographic status of HCWs, knowledge and perceptions of HCW on pediatric emergency triage, factors associated with quality of pediatric emergency triage as to HCWs perspective, and observation checklists for facility visits. Data collectors and supervisors with a nursing background were hired and given four days training on data collection techniques and study objectives.
The triage material and physical assessment were done via the use of a checklist on basic triage equipment, medicines and consumables (glucometer, IO needle, IV /rectal diazepam) as well as triage assessment forms, triage guidelines, sick child flow charts, the presence of a separate triage area for children and whether or not pediatric-specific treatment algorithms were present.

\subsection{Data Quality Management}

The data were coded, edited and entered into Epi-data version 3.01, cleaned and analyzed by Statistical software package for Social Science (SPSS) for windows version 20. Each completed questionnaire was checked for errors, completeness, and legibility immediately and missing or unclear data regained from the participant at the time. Filled questionnaires were stored safely with the principal researcher. Pre-coded data was directly entered onto a computer file to create a data set. For questions with the possibility of more than one response, each response was coded as a separate question and codes were assigned to the responses. Data from open-ended questions and other unstructured formats were coded after reviewing.

\subsection{Data Analysis}

Descriptive statistics such as mean, median and standard deviations were done as appropriate. Frequency distribution and percentages were employed for categorical variables. Data analysis was accomplished with SPSS version 20.0. Frequencies and percentages were used on responses about knowledge on principles of pediatric emergency triage and to analyze data on factors associated with quality of pediatric emergency triage. For the open-ended questions, the principal investigator first read the responses on questionnaires and came up with key codes and themes during analysis. Then, the themes were used to come up with frequencies and percentages.

A multivariable logistic regression model was used for the variables having an association $(\mathrm{P}<0.25)$ in the bivariate logistic regression model. Finally, it was evaluated that variables identified as associated $(\mathrm{P}<0.05)$ with the outcome variable in the multivariable analysis were used to predict the existence of the statistically significant association.

\subsection{Ethical Issues}

Ethical clearance was obtained from Addis Ababa University, School of Allied Health Sciences Institutional Review Board. The study was commenced after the letter of cooperation written to Hospitals from Zonal Health department. Written informed consent was obtained from the respective participants before participation in the study.

\subsection{Operational Definitions}

Good Quality Pediatric emergency triage: is present if an immediate categorization of a child with emergency or priority signs without any delay, adherence to the national guideline, having high-level triage knowledge and confidence of HCWs and availability of basic triage infrastructures. 
Poor quality of pediatric emergency triage: delay in child triage, no adherence to national guidelines, and low level of triage knowledge, low confidence and lack of basic triage infrastructure.

High-level confidence: if $80-100 \%$ of HCWs feel not confident when assigned in pediatric emergency triage.

Triage knowledge is the awareness of the health care workers about the key principles related to pediatric emergency triage and it was measured in the following way:

$\begin{array}{ll}\text { Percent }(\%) & \text { Level } \\ 80-100 & \text { High } \\ 60-79 & \text { medium } \\ \text { Less than } 60 & \text { Low }\end{array}$

Adherence to guidelines: Conformity in fulfilling or following officially recognized clinical practice guidelines as to ETAT+ Ethiopia.

Availability of essential drugs, equipment and lab. tests: At least 2 of equipment's listed, 2 drugs listed and blood glucose test is available

\section{Results}

\subsection{Socio-demographic Characteristics}

A total of 175 health care workers participated in the survey making the response rate of $98.31 \%$. Most of the respondents $108(61.7 \%)$ were male and $115(67.5 \%)$ were nurses in their category of profession. The majority of participants $113(64.6 \%)$ were aged between 20-30 years with a mean age of $30.38+5.2$ years. The study revealed that $99(56.6 \%)$ of the respondents were Protestant and 125 (71.4\%) of respondents were Wolaita ethnic group. The study showed that $46(26.3 .1 \%)$ were degree nurses, $73(41.7 \%)$ were diploma nurses, $23(13.1 \%)$ were health officers, 15 $(8.6 \%)$ were midwives, $13(7.3 \%)$ were General practitioner, $3(1.7 \%)$ were pediatricians and $11(6.8 \%)$ of respondents were others (internists, MSc nurses and MSc in emergency obstetrics) in their level of qualification (See Table1).
Table 1. Socio-demographic characteristics of health care professionals (respondents) in selected hospitals of Wolaita Zone, Southern Ethiopia, 2017.

\begin{tabular}{|c|c|}
\hline Socio-Demographic Variable & Frequency $(n=175, \%)$ \\
\hline \multicolumn{2}{|l|}{ Age group } \\
\hline $20-30$ & $113(64.57)$ \\
\hline $31-40$ & $56(32.00)$ \\
\hline $41-50$ & $5(2.85)$ \\
\hline $51+$ & $1(0.57)$ \\
\hline \multicolumn{2}{|l|}{ Sex of HCW } \\
\hline Male & $108(61.7)$ \\
\hline Female & $67(38.3)$ \\
\hline \multicolumn{2}{|l|}{ Ethnicity } \\
\hline Wolaita & $125(71.4)$ \\
\hline Amhara & $20(11.4)$ \\
\hline Others & $30(17.2)$ \\
\hline \multicolumn{2}{|l|}{ Religion } \\
\hline Protestant Christians & $99(56.6)$ \\
\hline Orthodox Christians & $52(29.7)$ \\
\hline Catholic & $16(9.1)$ \\
\hline Others & $8(4.6)$ \\
\hline \multicolumn{2}{|l|}{ Category of profession } \\
\hline medical doctor & $18(10.3)$ \\
\hline nurse & $115(65.7)$ \\
\hline midwife & $15(8.6)$ \\
\hline Health officer & $23(13.1)$ \\
\hline specialist & $4(2.3)$ \\
\hline \multicolumn{2}{|l|}{ level of qualification } \\
\hline diploma nurse & $73(41.71)$ \\
\hline $\mathrm{BSc}$ & $75(42.85)$ \\
\hline GP & $13(7.42)$ \\
\hline Pediatricians & $3(1.71)$ \\
\hline others & $11(6.28)$ \\
\hline
\end{tabular}

\subsection{Experience of $\mathrm{HCWs}$}

This study showed that $74(42.3 \%, n=175)$ of HCWs have experience of less than five years, $80(45.7 \%)$ of the health care workers included in the study have experienced of 6-10 years in the health facilities and $21(12 \%)$ have experienced greater than 10 years. (See Figure 1).

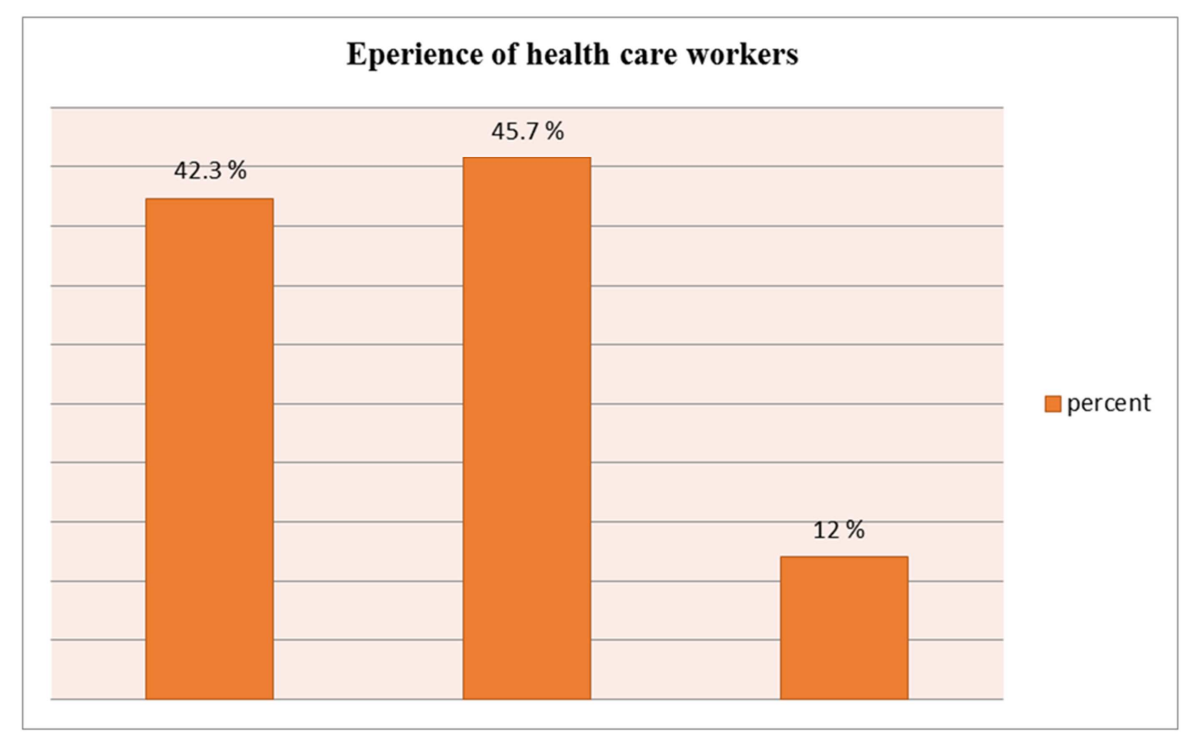

Figure 1. Experience of HCWs on selected hospitals of Wolaita zone. 


\subsection{Triage Knowledge of $\mathrm{HCW}$}

The majority 102 (58.3\%) of the respondents have low level knowledge on the triage definition, $33(18.9 \%)$ recognized triage duration and $25(14.28 \%)$ respondents were able to identify all triage places. Fifty-seven (32.57\%) of HCWs were able to categorize child with the emergent condition. Solely 63 $(36 \%)$ were able to categorize the child with urgent signs which need immediate attention. (See Table 2).

Table 2. Triage knowledge level of health care workers on selected hospitals.

\begin{tabular}{llll}
\hline Items & Low level knowledge (<60\%) & Medium level knowledge (60-79\%) & High level knowledge (>80\%) \\
\hline Triage definition & $102(58.3)$ & 0.00 & 0.00 \\
Triage duration (15-20 sec.) & $33(18.9)$ & 0.00 & 0.00 \\
All triage place (OPD, ward \& ER) & $25(14.28)$ & 0.00 & 0.00 \\
What are emergency signs & $57(32.57)$ & 0.00 & 0.00 \\
What are urgent signs & $63(36)$ & 0.00 & 0.00 \\
\hline
\end{tabular}

\subsection{Type of Training Taken by HCWs}

The present study shown that 140 (79.99\%) of the respondents have taken different kinds of training, of which, only $16(9.1 \%)$ of HCWs have adult Emergency triage assessment and Treatment (ETAT) training, 31 (17.7\%) have Integrated Management of Newborn and childhood Illness
(IMNCI) 16 (9.1\%) have resuscitation training, the majority of them were trained in severe acute malnutrition 40 (22.9\%), $31(17.7 \%)$ have training experience in infection prevention, $6(3.4 \%)$ were trained in others and $35(20 \%)$ of HCWs have no training experience at all. Others include malaria training, food in prescription and intensive care unit case management. (See Figure 2).

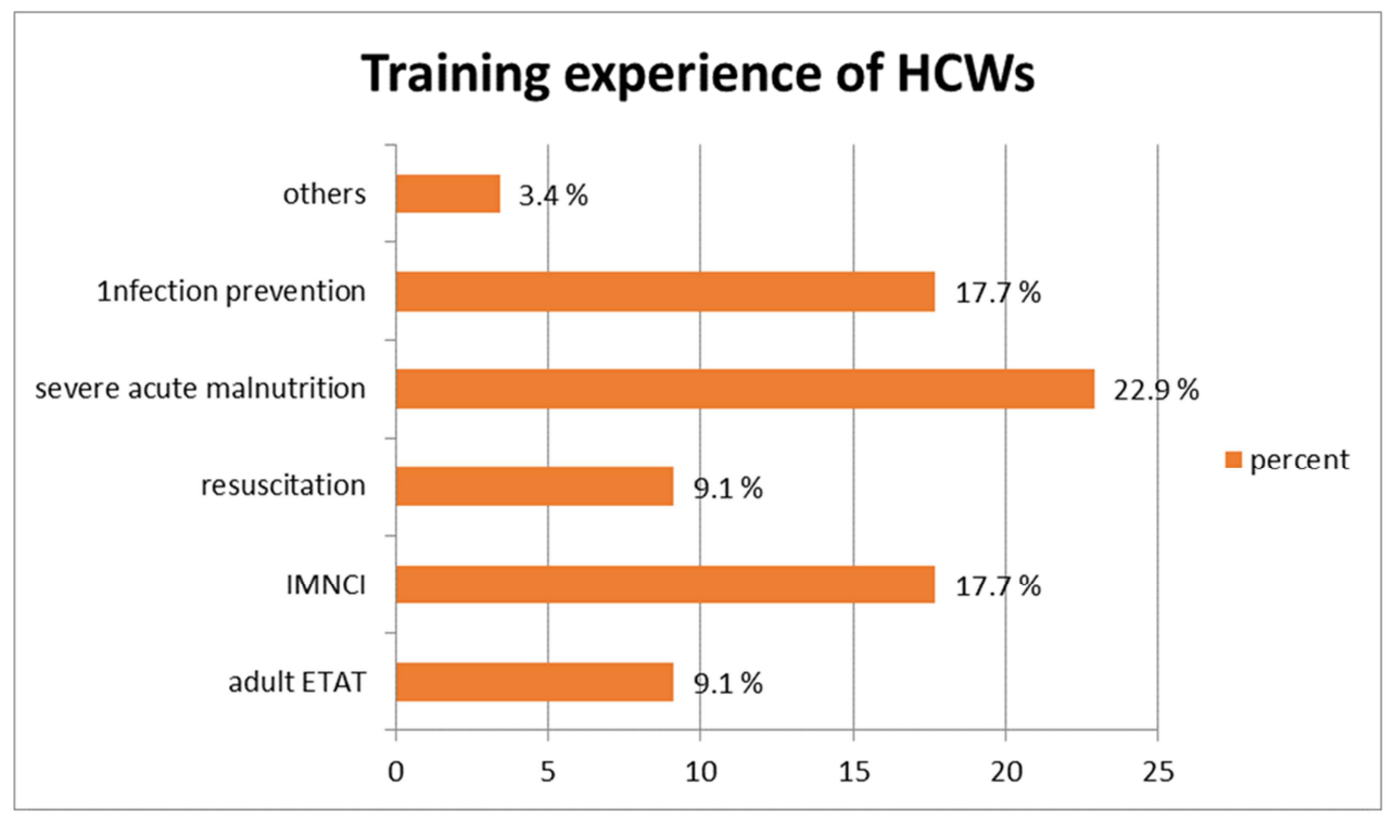

Figure 2. Shows type of training taken by HCWs on the studied hospitals.

\subsection{HCWs Responsible for Pediatric Emergency Triage}

The majority $109(62.3 \%)$ of the respondents stated that pediatric emergency triage should be done by pediatricians, $56(32 \%)$ reported that it should be carried out by medical doctors and $10(5.8 \%)$ suggested that it is the responsibility of the pediatric nurses. (See Table 3).

Table 3. Showing distribution of HCWs responsible for pediatric emergency triage as respondents answered.

\begin{tabular}{lll}
\hline Who is responsible? & Frequency $(\mathbf{n = 1 7 5})$ & Percentage $(\mathbf{\%})$ \\
\hline Paediatrician & 109 & 62.28 \\
Medical doctor & 56 & 32.00 \\
Pediatric nurse & 10 & 5.72 \\
\hline
\end{tabular}

\subsection{Perception of HCWs Towards Pediatric Emergency Triage}

Regarding feeling of health care providers towards pediatric emergency triage, $166(94.9 \%)$ reported that triage increases quality, 37 (21.1\%) said that it did not need highly qualified professionals, $79(45.1 \%)$ stated that it did not need expensive instrument, $29(16.6 \%)$ reported that pediatric triaging does not rationalize treatment, 169 (96.6\%) declared that it decreases early mortality and $17(9.7 \%)$ stated that it decreases length of stay. (See Table 4). 
Table 4. HCWs perception towards pediatric emergency triage.

\begin{tabular}{lll}
\hline Items & Yes & no \\
\hline Pediatric emergency triage increases the quality of care & $166(94.9 \%)$ & $9(5.1 \%)$ \\
Triaging of a sick child needs highly qualified health professionals & $138(78.9 \%)$ & $37(21.1 \%)$ \\
Triaging of a sick child needs an expensive instrument & $96(54.9 \%)$ & $79(45.1 \%)$ \\
Triaging of a sick child increases rational treatment of cases & $146(83.4 \%)$ & $29(16.6 \%)$ \\
Triaging of a sick decrease early mortality of children & $169(96.6 \%)$ & $6(3.4 \%)$ \\
Triaging of a sick child decreases the length of hospital stay? & $158(90.3 \%)$ & $17(9.7 \%)$ \\
\hline
\end{tabular}

\subsection{Observation Findings}

\section{Availability of Resource and Structural Qualities}

The observation was done focusing on the availability of resources needed to perform pediatric emergency triage, and the structural qualities of the selected facilities. All the health facilities have active triage systems and treatment area for emergency cases in the emergency room. Regarding necessary equipment's, all selected units were equipped with a functioning oxygen cylinder, fluids and drugs desired for emergency triage including salbutamol puffers, adrenaline, and diazepam IV and all kinds of IV fluids critical in the unit were obtainable. But only one hospital (Otona teaching and referral hospital) has separate triage area or emergency room for children however the other two hospitals (Christian general and Dubo Saint Mary catholic district hospitals) have no child appropriate triage space and they provide the service together with adult cases. None of the health facilities have had to work ETAT+ Ethiopia guidelines, ETAT + Ethiopia charts/formats, have had no sick child flow charts as of cardiopulmonary resuscitation, neonatal resuscitation and emergency management of triaged children. There are no treatment algorithms on child emergency settings which include asthma, anaphylaxis, and DKA and pain in all health facilities. Only teaching and referral hospital has sick child flow charts on severe dehydration. None of the hospitals have IO needle. Except in general hospital, there was only one oxygen cylinder for 6-8 patients in the units. The hospitals included in the study were lacking glucometer, and hemoglobin as essential laboratory support in the unit, however, they access it from the hospital main laboratory. This finding is also supplemented with the providers' perspective in which they mentioned the absence of ETAT + Ethiopia clinical management or practice guideline and lack of familiarity with the guidelines as one of the barriers for the provision of good pediatric emergency triage service.

All the selected facilities have consumable materials. All hospitals included in the study did not triage soon after arrival but did it after registration has been done which might result in a delay of assessment or treatment. Health care professionals in all selected hospitals were not using ETAT+ guideline. (See Table 5).

Table 5. Availability of resources and structural qualities of selected hospitals.

\begin{tabular}{|c|c|c|c|}
\hline Items & Teaching and referral Hosp & General Hosp & District Hosp \\
\hline \multicolumn{4}{|l|}{ 1. Availability of clinical guidelines } \\
\hline Availability of ETAT + guidelines & No & no & No \\
\hline Availability of ETAT + chart & No & no & No \\
\hline Availability of treatment algorithm for emergency conditions & No & no & no \\
\hline Adherence to guidelines & No & no & no \\
\hline \multicolumn{4}{|l|}{ 2. Equipments and consumables } \\
\hline Availability of essential medicines & Yes & yes & Yes \\
\hline \multicolumn{4}{|l|}{ 3. Structural qualities } \\
\hline Separate triage area for children & Yes & no & No \\
\hline Room for emergency treatment & Yes & yes & Yes \\
\hline Patients triaged without any delay & No & no & No \\
\hline Availability of essential lab tests in unit & no & no & no \\
\hline Training on pediatric emergency triage assessment and treatment guideline & no & no & no \\
\hline Training on critical illness including trauma & no & no & no \\
\hline
\end{tabular}

\subsection{Provider Perspective on Quality of Pediatric Emergency Triage Service}

One hundred forty one $(80.6 \%)$ of HCWs stated that work overload affects quality care. The frequently reported problems were lack of availability of ETAT + guidelines 150 $(85.7 \%)$ and absence of ETAT training $150(85.7 \%)$, lack of familiarity with guidelines $152(86.9 \%)$, lack of support 136 (77.7\%), lack of protocols and standards 139 (79.9\%), absence of separate triage or emergency area for children 148 $(84.6 \%)$, poor communication among staff $115(65.7 \%)$ and overcrowding $145(82.9 \%)$ are factors affecting pediatric triage quality as responded by $\mathrm{HCWs}$ in their health facilities. (See Table 6). 
Table 6. Factors affecting triage quality as to respondents.

\begin{tabular}{lll}
\hline Variables & Yes (\%) & No (\%) \\
\hline work overloads & $141(80.6)$ & $34(19.4)$ \\
Lack of availability of pediatric ETAT guideline & $150(85.7)$ & $25(14.3)$ \\
The absence of ETAT training & $161(92)$ & $14(8)$ \\
Lack of familiarity with ETAT guidelines & $152(86.9)$ & $23(13.1)$ \\
lack of support from hospital management & $136(77.7)$ & $39(22.3)$ \\
Lack of protocols standards & $139(79.9)$ & $36(20.6)$ \\
No separate triage or emergency area for children & $148(84.6)$ & $27(15.4)$ \\
Poor communication among staff & $115(65.7)$ & $60(34.3)$ \\
overcrowding of emergency room & $145(82.9)$ & $30(17.1)$ \\
\hline
\end{tabular}

\subsection{The Response of HCWs on Emergent Signs Among Studied Hospitals}

The present study has shown that $31(17.7 \%)$ of the respondents identified central cyanosis as emergency signs and $12(6.9 \%)$ stated that absent breathing as one of the signs for immediate treatment, $14(8 \%)$ did not identify circulation problem, 14 not recognized coma or conscious, 23 (13.1\%), $14(10.3 \%), 18(10.3 \%), 31(17.7 \%)$ did not identify child with the signs of convulsion, severe dehydration, bleeding, open fracture child as emergent categorization respectively. (Table 7).

Table 7. Showing the response of health care workers on emergent signs.

\begin{tabular}{lll}
\hline Emergent signs & Yes (\%) & No (\%) \\
\hline Central cyanosis & $144(82.3)$ & $31(17.7)$ \\
Obstructed/absent breathing & $163(93.1)$ & $12(6.9)$ \\
Circulation problem & $161(92)$ & $14(8)$ \\
Coma or unconscious & $161(92)$ & $14(8)$ \\
Convulsion & $152(86.9)$ & $23(13.1)$ \\
Severe dehydration & $161(92)$ & $14(8)$ \\
Bleeding & $157(89.7)$ & $18(10.3)$ \\
Open fracture & $141(81.7)$ & $31(17.7)$ \\
\hline
\end{tabular}

\subsection{The Response of HCWs on Urgent Signs Among Studied Hospitals}

From the study participants, 33 (18.9\%), 21 (12\%), and 15 $(8.6 \%)$ of health care providers did not identify severe pallor, history of poisoning and severe pain as urgent sign respectively. $78(44.6 \%), 72(41.1 \%), 32(18.3 \%), 14(8 \%)$, $11(6.3 \%), 31(17.7 \%)$ and $33(8.9 \%)$ of health care professionals were not categorized children with edema of both feet, severe visible wasting, urgent referral child, burn, respiratory distress, any sick child less than two months, and a child with very hot or cold in their category of classification respectively. (See Table 8).

Table 8. Response of HCWs to urgent signs.

\begin{tabular}{lll}
\hline Urgent signs & Yes (\%) & No (\%) \\
\hline Severe pallor & $142(81.1)$ & $33(18.9)$ \\
History of poisoning & $154(88)$ & $21(12)$ \\
Severe pain & $160(91.4)$ & $15(8.6)$ \\
edema of both feet & $97(55.4)$ & $78(44.6)$ \\
Severe visible wasting & $103(58.9)$ & $72(41.1)$ \\
Urgent referral & $143(81.7)$ & $32(18.3)$ \\
Burn & $161(92)$ & $14(8)$ \\
Respiratory distress & $164(93.7)$ & $11(6.3)$ \\
Any Sick child less than 2 months & $144(82.3)$ & $31(17.7)$ \\
A very hot or cold child & $142(81.1)$ & $33(8.9)$ \\
\hline
\end{tabular}

As responded by $\mathrm{HCWs}$, there was a lack of awareness and commitment amongst health care workers. HCWs said that essential equipment's especially oxygen cylinder and lab support were lacking in their health facilities.

Variables significantly associated with quality of pediatric emergency triage.

Table 9. Shows factors associated with quality of pediatric emergency triage by the multivariate logistic regression model.

\begin{tabular}{|c|c|c|c|c|c|c|}
\hline \multicolumn{7}{|c|}{ Quality of pediatric emergency triage } \\
\hline Variable & Fre. $(\%)$ & P. value & Good quality & Poor quality & Crude OR (CI) & AOR (CI) \\
\hline $\begin{array}{l}\text { Level of qualification MSc } \\
\text { Read guideline }\end{array}$ & $9(5.1)$ & 0.010 & $9(5.1)$ & 0.000 & $0.815(0.519-1.281)$ & $0.021(0.001-0.398)$ \\
\hline $\begin{array}{l}\text { Yes } \\
\text { No }\end{array}$ & $\begin{array}{l}123(70.3) \\
52(29.7)\end{array}$ & 0.023 & $\begin{array}{l}102(58.28) \\
31(17.71)\end{array}$ & $\begin{array}{l}21(12) \\
73(41.71)\end{array}$ & $\begin{array}{l}1 \\
3.164(0.673-2.489)\end{array}$ & $2.807(1.139-6.926)$ \\
\hline Training & & & & & & \\
\hline $\begin{array}{l}\text { yes } \\
\text { no }\end{array}$ & $\begin{array}{l}98(56) \\
77(44)\end{array}$ & $\begin{array}{l}1 \\
0.029\end{array}$ & $\begin{array}{l}117(66.85) \\
29(16.57)\end{array}$ & $\begin{array}{l}29(16.57) \\
48(27.42)\end{array}$ & $\begin{array}{l}1 \\
2.687(0.365-1.240)\end{array}$ & $5.847(1.197-28.850)$ \\
\hline
\end{tabular}

Reading guidelines, having training and level of qualification of health care workers at $P$. value $<0.025$. The likely hood of triage quality of HCWs who read guidelines 2.807 times more than that of that of caregivers who did not read guidelines $(\mathrm{AOR}=2.807,95 \% \mathrm{CI}=1.139-6.926)$. The likely hood of having pediatric emergency triage quality is 5.847 times higher in trained health care professionals than that of HCWs who were not trained $(\mathrm{AOR}=5.847$,
95CI=1.197-2.850) (See Table 9).

\section{Discussion}

\subsection{Qualities of Pediatric Emergency Triage Structural Qualities}

Similar with study in other resource-limited countries $[1,3$, 
16], this investigation identified that quality of care on pediatric emergency triage in selected hospitals was found to be poor and needs upgrading. The deficits acknowledged were found in a number of interrelated parts including knowledge and feeling of health care workers, standardized tools, protocols, clinical practice guidelines, sick child flow charts, crucial lab support, vital hospital support systems, equipment's, drugs and child appropriate triage.

As to this study, $115(65.7 \%)$ of respondents in surveyed health facilities were nurses in their category of profession. This is in line with the study in Rwanda on quality of hospital care for children where nurse were 110 (64.4\%). This might be due to the similar infrastructure of the two countries. This study reported that $22(12.6 \%)$ of HCWs were physicians in their profession. This is slightly different from the study in Rwanda on quality of hospital care for children where 37 $(21.6 \%, n=171)$ [17] were physicians. This might be due to better provision of incentives to physicians or great attention of hospitals on quality of pediatric care.

The present study revealed that 159 (90.9\%) of respondents were providing pediatric emergency service without specific or related training. This is higher than the study in Tanzania, Australia, and Iran, where 78\%, 42\% and $14 \%$ of the nurses working in the emergency centers provide care without formal training in emergency care or triage respectively. This leads to mistaken triage decisions as knowledge on triage has been recognized as a decisive issue that powers exactness of triage decision and lasts in poor quality $[18,19]$. This might be due to a lack of sustainable development for clinicians on pediatric emergencies or poor attention was paid to the care from hospital managers, or absence of monitoring from local or national government officials, staffs were not qualified in pediatric emergencies, concentration of hospitals on chief financial or high-tech investment rather than a better use of existing resources (protocols and guidelines) to improve quality of care.

This study showed that health care providers have had low-level knowledge on triage definition, triage duration, all triage place, were unable to categorize emergent or urgent cases and none of them identified clinical signs as triage criteria. This finding is in line with the study done in Tanzania and USA $[18,20]$. This may lead to deadly delay or under-triage which will result in lower acuity level than required. The consequence will be wastage of ED resources, lengthy waiting times for patients to receive care, and development of dangerous complications which will preventable if correct identification [2, 3, 21]. This might be due to an absence of training to the staffs on pediatric emergency triage assessment and treatment who are working in peripheral hospitals of the countries or lack of online information.

\subsection{The Feeling of Health Care Workers}

This study showed that health care providers were frustrated and uninterested when allocated in pediatric emergency triage. This might indicate that they have had a low level of confidence and results in too slow recognition of critical cases. This is congruent to the study in the USA which directed that HCWs in pediatric emergency feel uncomfortable [20]. This might be due to a lack of sustainable staff development, lack of in-service training or lack of onsite mentoring from the senior staffs.

According to this study finding, health care professionals since that pediatric emergency triage needs highly qualified caregivers or expensive instruments which contradicts with the findings of many resource-limited countries such as Uganda, Gahanna, Rwanda, and Kenya [22-24]. The feeling expressed by health workers on the surveyed may be due to lack of awareness or misconception on principles of pediatric emergency triage or due to the perception that pediatric emergency triage needs to be complicated and technologically sophisticated. This, in turn, will results in poor quality care, delay in treatment. As consistent to the other research in middle and low-income countries, this study found that pediatric emergency triage could have the capability to decrease noticeably pediatric illness, LOS and death as described by health care providers, hence can increase the quality of emergency care for hospitalized children $[46,48]$.

\subsection{Availability of Resources}

Based on this assessment, all of the health facilities studied has active triage system and basic equipment's needed for pediatric emergency triage. But only one hospital has a separate triage area for children (Otona teaching and referral hospital). This is congruent to the reports from Amman, Jordan [49]. This might be due to the negligence of higher officials on the importance of pediatric emergency conditions including triage. This would make identification of pediatric emergencies or urgencies unlikely and can delay care which can result in a poor patient outcome. None of the hospitals have glucometer on their emergencies or pediatric units. This might be due to insufficient logistic supply and also the absence of emphasis on identification of cause for convulsion. This could result in less likely recognition of reasons for convulsing child. The absence of such a test has a clear effect on the diagnosis of hypoglycemia. All hospitals have had no clinical practice guidelines including ETAT + Ethiopia, sick child flow charts and treatment algorithm on pediatric emergencies. This might be due to reluctance to change from hospital administrators side or caregivers side, knowledge deficit, and leadership problems from hospitals, not using CPGLs as a requirement in pediatrics department, absence of guidelines at national level or pooling of concentration at academic or tertiary level hospitals from concerned bodies, or paying little attention to hospitals in peripheral levels This finding is similar to the study done in Rwanda and contradicting to the research in Tanzania which has shown that one of emergency center from four hospitals surveyed have ETAT and pain assessment guideline. [42, 50].

It is clear that nonexistence of such clinical practice guidelines leads to confusion of HCWs on the identification of emergent or urgent cases which result in a delay of treatment, increases the length of hospital stay, lasts in 
avoidable death or preventable complication and ends in an unnecessary referral. The final is the poor quality of pediatric emergency care including triage. This finding is also supplemented with the providers' perspective in which the health care professionals declared that absence of guideline as one of the obstacle to provide good quality of pediatric emergency care including triage.

This study pointed out that there is a major gap in the equipment necessary to provide pediatric emergency triage service which compromises quality. Except for one hospital, there is a high shortage of oxygen cylinder or concentrator for management of severe respiratory distress. All surveyed hospitals have no intra-osseous needle. This might make management of severe dehydration or shock very difficult. This might be due to inadequate supply from hospitals or lack of awareness amongst emergency departments. This finding is similar to the research done in Rwanda and other developing countries like Kenya and Tanzania which identified major gaps in resource availability (e.g., 50\% of hospitals without intra-osseous needles for the management of shock in all hospitals surveyed, no nebulizer in $25 \%$ of the hospitals $[4,50]$. In this study, for example, two of the hospitals use one oxygen cylinder for 6-8 children (Otona teaching and referral and Dubo Saint Mary catholic district hospital). Health care professional in all three hospitals was not adhering to guidelines (no adherence) when triaging a child with emergent or urgent signs. This might be due to the absence of guidelines in the selected hospitals or due to the absence of training on ETAT + Ethiopia. This may result in the missed categorization of the child. This finding contradicting to the study in Kabul, Afghanistan, where adherence to ETAT+ guideline was reported as low in a different place and high in an only one site [49].

This study demonstrated that all hospitals surveyed have no glucometer, were lacking oxygen cylinder, have no essential laboratory support in their unit and two of three hospitals have no separate child triage area. This is similar to the study in Bangladesh which indicated that hospitals were lacking essential laboratory support and equipment's [51]. This shows the quality of care for children in the studied hospitals was poor and needs improvement.

As responded by HCWs, there was a lack of awareness and commitment amongst health care workers. HCWs said that essential equipment's especially oxygen cylinder and lab support were lacking in their health facilities. This finding is in line with a study in Iran which reported that physical structure of the health care facilities and lack of equipment was factors of triage quality (6). Among the various things related to their working situation, service providers revealed that lack of pediatric emergency triage specific training and onsite orientation, allocation of service providers based on their previous experience rather than related training, inadequacy of drugs and material supply, lack of motivation and absence of standardized triage tools were the key factors that can avert the provision of worthy quality of pediatric emergency triage. This perspective is similar to the finding from an observation checklist. This shows that quality of pediatric emergency is suboptimal in the hospitals studied. This finding is congruent to the study in Hull University, United Kingdom, which suggested that working rapport within the team and within the hospital, the professional atmosphere and hierarchal communication affects triage quality [15].

\subsection{Factors Affecting the Quality of Pediatric Emergency Triage}

This finding has shown that there was a slight positive association between pediatric emergency triage quality and training ( $\mathrm{P}-\mathrm{Value}=0.056$ ). This might be due to exposure of health care providers to update guidelines. This is similar to the study in Kenya [52].

This finding revealed that reading of pediatric emergency triage guideline was significantly associated with pediatric emergency triage quality $(\mathrm{AOR}=2.807,95 \% \mathrm{CI}=1.139-6.926$, $\mathrm{PV}=0.023$ ). This might be due to exposure of health care workers to up-to-date clinical practice or evidence-based guidelines and standards during reading and information sharing when they read guidelines. This result is congruent to the study in Iran [5].

The recent study suggested that there was an association between training experience and quality of pediatric emergency $(\mathrm{AOR}=5.847,95 \% \mathrm{CI}=1.197-2.850, \mathrm{PV}=0.029)$. This might be due to continuous educational development and gain of knowledge necessary for the accomplishment of pediatric emergency triage quality or this might be due to the training of health care professionals equips with the ability of identification of the children with emergency signs or priority signs without delay to take treatment or to be seen immediately. This is in line with the study done in Ghana [53].

This study has shown that there was no significant association between pediatric emergency triage quality and the experience of health care workers. This is contradictory to the research in Iran which showed experience as the supreme imperative factor that can influence triage decision making among health care professionals [5]. This might be due to the allocation of health care providers in pediatric emergency triage without training on up to date clinical practice guidelines or absence of continuous professional development in the hospitals studied or it may be due to under publication of the study with the negative finding.

\section{Conclusion}

This study indicated that overall quality of pediatric emergency triage service was poor and needs improvement which may be described by scarcity of essential equipment's and laboratory tests, absence of adherence to national guidelines, absences of guidelines, absence of trainings, absence of standards, absence of protocols, absence of sick child flow charts and treatment algorithm, low level of HCWs knowledge on principles of triage, low confidence of HCWs in the unit, absence of regular supervision and monitoring and absence of child-appropriate triage. The 
quality of pediatric emergency triage can be enhanced by availing clinical practice guidelines, by training $\mathrm{HCWs}$ on ETAT + Ethiopia who was delivering care to children, by strengthening supportive supervision and designing simple strategies as of using CPG to upgrade quality care for hospitalized children.

\section{Recommendations}

This study points the need for an incorporated package of quality upgrading measures on pediatric emergency service including triage. Since pediatric emergency triage is one of the strategies to enhance the quality of hospitalized care for children, the following suggestions were indicated.

\section{To Hospitals}

The hospital should avail essential drugs, equipment, CPGLs and laboratory supports as needed for health care providers to optimize the quality of care

Ongoing training, supportive supervision, and monitoring should be in place to motivate staffs and to feel knowledge gap thereby advance the service quality.

The hospital should improve infrastructure to ensure child appropriate triage service and should impress HCWs to use CPGLs as a requirement in pediatric departments

There should be continuous evaluation of $\mathrm{HCWs}$ on pediatric emergency unit ensure its quality.

The administrators of hospitals need to develop a formal pediatric emergency triage based training to staffs of the pediatric department in service level

\section{To Health Care Professionals}

Health care providers should pay great attention to pediatric emergency triage service quality and update them to contemporary ED service or should be trained in principles of pediatric emergency triage assessment and treatment to deliver qualified care to their clients

HCWs should integrate the simple and appropriate CPG for categorization of pediatric emergencies to enhance the quality of care for pediatric emergency

\section{To Researchers}

Researchers should give attention to the importance of pediatric emergency triage service since there is serious research gap in the area mentioned.

Statistics are on demand to allow continuous monitoring and assessment of the services provided and their sustainability.

To FDRE Minister of Health

The federal minister of health should set benchmarking or accreditations not only at the central level but also to the peripheral hospitals of the country to increase or to create a sense of competition among hospitals.

FDRE minister of health should work collaboratively with the minister of education to incorporate pediatric emergency triage assessment and treatment course in the undergraduate curriculum for health care professionals in the pre-service training program as sustainable quality improvement strategies for pediatric emergency

FDRE minister of health should collaborate and coordinate with Ethiopian pediatric and nursing association to provide training on pediatric emergency triage assessment and treatment for staffs working in pediatric departments of hospitals.

FDRE minister of health should pay attention to ensure appropriate dissemination of update guidelines to peripheral hospitals to enhance service quality.

\section{The Study Limitations}

One of the limitations of this study was that assessing only hospitals with established triage system while excluding others.

This survey focused on structural domains of quality measurement of health care services, the actual care of individual children is not seen.

The basic equipment's and drugs which are not availed at the time of auditing were taken as absent while it may be in the stock.

\section{Abbreviation and Acronyms}

CI
CPGLs
DKA
EDs
ETAT+ Ethiopia
FDRE
HCWs
IMNCI
IO
LOS
MOH
SNNPR
SPSS
UNICEF
WHO

\author{
Confidence Interval \\ Clinical Practice Guide Lines \\ Diabetic Keto acidosis \\ Emergency Departments \\ Emergency Triage assessment and Treatment plus admission \\ Federal Democratic Republic of Ethiopia \\ Health Care Workers \\ Integrated Management of Newborn and childhood illness \\ Intra-Osseous \\ Length of stay \\ Ministry of Health \\ South Nations Nationalities and Peoples Region \\ Statistical Package for Social Science \\ United Nations Children Education Fund \\ World Health Organization
}




\section{Competing Interests}

The authors have declared that no competing interests exist.

\section{Acknowledgements}

We would like to forward our gratitude to Addis Ababa University, School of Allied Health Sciences. We also thank Wolaita Zone administrators, the supervisors, respondents and Data Collectors.

\section{References}

[1] Hasan Rajabi MS, Gholamreza Khademi. Emergency Management of Common Diseases in Children. Int J Pediatr, Vol3, Serial No19, Jun 22, 2015; Vol. 3 (N. 4-1): 789-98). Epub jun 22, 2015.

[2] Harry Campbell TD, Martin Weberc, Mike English et al. Global Initiatives for Improving Hospital Care for Children: State of the Art and Future Prospects. 2007.

[3] Baker T. Critical care in low-income countries. Tropical Medicine and International Health. February 2009; volume 14 no 2 (no. 2): pp 143-8.

[4] Harmesh S. Bains RKS. A Simple Clinical Score "TOPRS" to Predict Outcome in Pediatric Emergency Department in a Teaching Hospital in India. Iran J Pediatr. 2012; Vol 22 (1): 97-101. Epub Jan 03, 2012.

[5] WHO. Facilitator guide: Triage and Emergency Treatments: Quick Check Essentials. 2014. Epub june 2014.

[6] Abbas Dadashzadeh FA, Azad Rahmani, Morteza Ghojazadeh. Factors affecting triage decision-making from the viewpoints of emergency department staff in Tabriz hospitals. Iran J Crit Care Nurs. 2014; vol. 6 (4): 261-6.

[7] Organization wh. Updated guidelines on Paediatric emergency triage, assessment and treatment Care of critically ill children. Updated guideline. 2016.

[8] K. Health FDRoEMoH. Emergency Triage Assessment and Treatment (ETAT + Ethiopia). 2014.

[9] Temmy Sunyoto RVdB, Pola Valles, Reinaldo Gutierrez, Latifa Ayada et al. Providing emergency care and assessing a patient triage system in a referral hospital in Somaliland: a cross-sectional study. BMC Health Services Research. 2014; 14: $1472-6963$.

[10] Augustyn JE. Aninvestigation into the implimentation of emergency unit triage system in a selected private hospital. 2006.

[11] Safari SR. Perceptions and challenges of emergency triage assessment and treatment guidelines in emergency departmenta at muhibilliI national hospital, Tanzania. 2012.

[12] Ling XJ. Risk factors on length of stay in pediatric emergency observation unit of a tertiary children's hospital in. August 2011.

[13] MEDICINE IFFE. Updated international standards of care for children in emergency departments. Version 20 June 2014. 2012.
[14] Baker et al. BMC Health Services Research 2013. Emergency and critical care services in Tanzania: a survey of ten hospitals. BMC Health Services Research. 2013; 13 (140).

[15] Acharya RP. Emergency department triage: an ethical analysis. BMC emergency medicine. 2011.

[16] Tamburlini T. Improving the quality of paediatric care in peripheral hospitals in developing countries. 563-5.

[17] Celestin Hategeka, Leah Mwai, and Lisine Tuyisenge. Implementing the Emergency Triage, Assessment and Treatment plus admission care $(\mathrm{ETAT}+)$ clinical practice guidelines to improve quality of hospital care in Rwandan district hospitals: healthcare workers' perspectives on relevance and challenges. 2017; 17 (256).

[18] Aloyce R. Assessment of knowledge and skills of triage amongst nurses working in the emergency centres in Dar es Salaam, Tanzania. African Journal of Emergency Medicine. 2013; 4: 14-8. Epub july, 2013.

[19] Rahmati H, Azmoon M, Kalantari Meibodi M, Zare N. Effects of Triage Education on Knowledge, Practice and Qualitative Index of Emergency Room Staff: A Quasi-Interventional Study. Bull Emergency Trauma. 2013; 1 (2): 69.

[20] Radesi L. Identifying Barriers to Successful Interventions for Pediatric Septic Found Shock Patients in Non-Pediatric Emergency Departments. Med Pub Journals. 2015; 7.

[21] Qureshi. Triage systems: a review of the literature with reference to Saudi Arabia. Eastern Mediterranean Health Journal. 2010; Vol. 16 (no. 6).

[22] David P South all BMP, Sue Wieteska, Martin P Samuels. Paediatric emergency care in resource-limited settings. April 2013; Vol 381.

[23] Paediatric Department CoM, Blantyre, Malawi. Triaging children. 2013; 103 (3).

[24] Heather L. Crouse etal. Impact of an Emergency Triage Assessment and Treatment (ETAT)-based triage process in the paediatric emergency department of a Guatemalan public hospital. Paediatric int child health. 2016; 36 (3): 219-24.

[25] Hansoti et al. Reliability and validity of pediatric triage tools evaluated in Low resource settings: a systematic review. BMC pediatrics. 2017; 17 (37).

[26] Bilir Ö. Perspectives of Emergency Department Staff on Triage Practice. Meandros Med Dent J 2017.18: 27-32. Epub 27.05.2016.

[27] H. Graham et al. Use of clinical guidelines: perspectives from clinicians in paediatric and maternity hospitals in Kabul, Afghanistan. Eastern Mediterranean Health Journal. 2015; 21 (2). Epub 04/12/14.=27.

[28] Hategeka C, Shoveller J, Tuyisenge L Kenyon C, Cechetto DF, Lynd LD. Pediatric emergency care capacity in a lowresource setting: An assessment of district hospitals in Rwanda. PLOS one doi: 10.1371/journal. Pone.0173233. March 3, 2017; 10.

[29] Hoque et al. An assessment of the quality of care for children in eighteen randomly selected district and subdistrict hospitals in Bangladesh. BMC Pediatrics. 2012; 12: 1471-2431. 
[30] Ouma N. Effect of an emergency care training on management of acute childhood diarrea in Nakuru district, KENYA. 2010
[31] Tette et al. Under-five mortality pattern and associated risk factors: a case-control study at the Princess Marie Louise Children's Hospital in Accra, Ghana. BMC pediatrics. 2016; 16 (148). 